\title{
Unifying Image Plane Liftings for Central Catadioptric and Dioptric Cameras
}

\author{
João P. Barreto ${ }^{12}$ and Kostas Daniilidis ${ }^{1}$ \\ 1 GRASP Laboratory, University of Pennsylvania, Philadelphia PA, 19104 \\ 2 ISR/DEEC, University of Coimbra, Coimbra, Portugal \\ \{jpbar, kostas\}egrasp.cis.upenn.edu
}

\begin{abstract}
In this paper, we study projection systems with a single viewpoint, including combinations of mirrors and lenses (catadioptric) as well as just lenses with or without radial distortion (dioptric systems). Firstly, we extend a wellknown unifying model for catadioptric systems to incorporate a class of dioptric systems with radial distortion. Secondly, we provide a new representation for the image planes of central systems. This representation is the lifting through a Veronese map of the original image plane to the 5D projective space. We study how a collineation in the original image plane can be transferred to a collineation in the lifted space and we find that the locus of the lifted points which correspond to projections of world lines is a plane in parabolic catadioptric systems and a hyperplane in case of radial lens distortion.
\end{abstract}

\section{Introduction}

A vision system has a single viewpoint if it measures the intensity of light travelling along rays which intersect in a single point in $3 \mathrm{D}$ (the projection center). Vision systems satisfying the single viewpoint constraint are called central projection systems. The perspective camera is an example of a central projection system. The mapping of points in the scene into points in the image is linear in homogeneous coordinates, and can be described by a $3 \times 4$ projection matrix $\mathbf{P}$ (pin-hole model). Perspective projection can be modeled by intersecting a plane with a pencil of lines going through the scene points and the projection center $\mathbf{O}$.

There are central projection systems whose geometry can not be described using the conventional pin-hole model. In [1] Baker et al. derive the entire class of catadioptric systems verifying the single viewpoint constraint. Sensors with a wide field of view and a unique projection center can be built by combining a hyperbolic mirror with a perspective camera, and a parabolic mirror with an orthographic camera (paracatadioptric system). However the mapping between points in the 3D world and points in the image is non-linear. In [12] it is shown that in general the central catadioptric projection of a line is a conic section. A unifying theory for central catadioptric systems has been proposed in [6]. It is proved that central catadioptric image formation is equivalent to a projective mapping from a sphere to a plane with a projection center on a sphere axis perpendicular to the plane. Perspective cameras with non-linear lens distortion are another example of central projection systems where the relation in homogeneous coordinates between scene points and image points is no longer linear. True lens distortion curves are typically very complex and higher-order models are introduced to approximate the distortion during calibration $[3,14]$. However, simpler low-order models can 


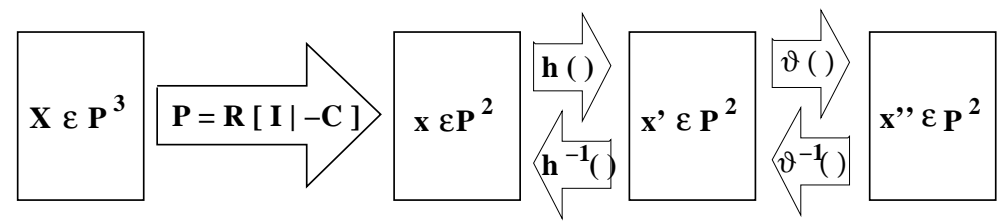

Fig. 1. Unifying Image Formation Model

be used for many computer vision applications where an accuracy in the order of a pixel is sufficient. In this paper the radial lens distortion is modeled after the division model proposed in [5]. The division model is not an approximation to the classical model in [3], but a different approximation to the true curve. In this paper, we present two main novel results:

1. The unifying model of central catadioptric systems proposed [6] can be extended to include radial distortions. It is proved, that the projection in perspective cameras with radial distortion is equivalent to a projective mapping from a paraboloid to a plane, orthogonal to the paraboloid's axis, and with projection center in the vertex of the paraboloid. It is also shown that, assuming the division model, the image of a line is in general a conic curve.

2. For both catadioptric and radially distorted dioptric systems, we establish a new representation through lifting of the image plane to a five-dimensional projective space. In this lifted space, a collineation in the original plane corresponds to a collineation of the lifted points. We know that world line project to conic sections whose representatives in the lifted space lie on a quadric. We prove that in the cases of parabolic catadioptric projection and radial lens distortion this quadric degenerates to a hyperplane.

\section{A Unifying Model for Perspective Cameras, Central Catadioptric Systems, and Lenses with Radial Distortion}

In [6], a unifying model for all central catadioptric systems is proposed where conventional perspective imaging appears as a particular case. This section reviews this image formation model as well as the result that in general the catadioptric image of a line is a conic section [12]. This framework can be easily extended to cameras with radial distortion where the division model [5] is used to describe the lens distortion.

This section shows that conventional perspective cameras, central catadioptric systems, and cameras with radial distortion underly one projection model. Fig. 1 is a scheme of the proposed unifying model for image formation. A point in the scene $\mathbf{X}$ is transformed into a point $\mathbf{x}$ by a conventional projection matrix $\mathbf{P}$. Vector $\mathbf{x}$ can be interpreted both as a 2D point expressed in homogeneous coordinates, and as a projective ray defined by points $\mathbf{X}$ and $\mathbf{O}$ (the projection center). Function $\hbar$ transforms $\mathbf{x}$ in the intermediate point $\mathbf{x}^{\prime}$. Point $\mathbf{x}^{\prime}$ is related with the final image point $\mathbf{x}^{\prime \prime}$ by function $\partial$. Both $\hbar$ and $\partial$ are transformations defined in the two dimensional oriented projective space. They can be linear or non-linear depending on the type of system, but they are always injective functions with an inverse. Tab. 1 summarizes the results derived along this section. 


\begin{tabular}{|c|c|}
\hline \multicolumn{2}{|l|}{ Perspective Camera $(\xi=0, \psi=0)$} \\
\hline$\hbar(\mathbf{x})=(x, y, z)^{t}$ & $\partial\left(\mathbf{x}^{\prime}\right)=\mathbf{K} \mathbf{x}^{\prime}$ \\
\hline$\hbar^{-1}\left(\mathbf{x}^{\prime}\right)=\left(x^{\prime}, y^{\prime}, z^{\prime}\right)^{t}$ & $\partial^{-1}\left(\mathbf{x}^{\prime \prime}\right)=\mathbf{K}^{-1} \mathbf{x}^{\prime \prime}$ \\
\hline \multicolumn{2}{|l|}{ Hyperbolic Mirror $(0<\xi<1)$} \\
\hline $\begin{array}{l}\hbar(\mathbf{x})=\left(x, y, z+\xi \sqrt{x^{2}+y^{2}+z^{2}}\right)^{t} \\
\hbar^{-1}\left(\mathbf{x}^{\prime}\right)=\left(x^{\prime}, y^{\prime}, z^{\prime}-\frac{\left(x^{\prime 2}+y^{\prime 2}+z^{\prime 2}\right) \xi}{z^{\prime} \xi+\sqrt{z^{\prime 2}+\left(1-\xi^{2}\right)\left(x^{\prime 2}+y^{\prime 2}\right)}}\right)\end{array}$ & $\begin{array}{l}\widetilde{\partial}\left(\mathbf{x}^{\prime}\right)=\mathbf{H}_{\mathbf{c}} \mathbf{x}^{\prime} \\
\check{\partial}^{-1}\left(\mathbf{x}^{\prime \prime}\right)=\mathbf{H}_{\mathbf{c}}^{-1} \mathbf{x}^{\prime \prime}\end{array}$ \\
\hline \multicolumn{2}{|l|}{ Parabolic Mirror $(\xi=1)$} \\
\hline $\begin{array}{l}\hbar(\mathbf{x})=\left(x, y, z+\sqrt{x^{2}+y^{2}+z^{2}}\right)^{t} \\
\hbar^{-1}\left(\mathbf{x}^{\prime}\right)=\left(2 x^{\prime} z^{\prime}, 2 y^{\prime} z^{\prime}, z^{\prime 2}-x^{\prime 2}-y^{\prime 2}\right)^{t}\end{array}$ & $\begin{array}{l}\partial\left(\mathbf{x}^{\prime}\right)=\mathbf{H}_{\mathbf{c}} \mathbf{x}^{\prime} \\
\partial^{-1}\left(\mathbf{x}^{\prime \prime}\right)=\mathbf{H}_{\mathbf{c}}{ }^{-1} \mathbf{x}^{\prime \prime}\end{array}$ \\
\hline \multicolumn{2}{|l|}{ Radial Distortion $(\xi<0)$} \\
\hline $\begin{array}{l}\delta\left(\mathbf{x}^{\prime}\right)=\left(2 x^{\prime}, 2 y^{\prime}, z^{\prime}+\sqrt{z^{\prime 2}-4 \xi\left(x^{\prime 2}+y^{\prime 2}\right)}\right)^{t}, \\
\delta^{-1}\left(\mathbf{x}^{\prime \prime}\right)=\left(x^{\prime \prime} z^{\prime \prime}, y^{\prime \prime} z^{\prime \prime}, z^{\prime \prime 2}+\xi\left(x^{\prime \prime 2}+y^{\prime \prime 2}\right)\right)^{t},\end{array}$ & $\begin{array}{l}\hbar(\mathbf{x})=\mathbf{K} \mathbf{x} \\
\hbar^{-1}\left(\mathbf{x}^{\prime}\right)=\mathbf{K}^{-1} \mathbf{x}^{\prime}\end{array}$ \\
\hline
\end{tabular}

Table 1. The mapping functions $\hbar$ and $\widetilde{\partial}$ and corresponding inverses

\subsection{Perspective Camera and Central Catadioptric Systems}

The image formation in central catadioptric systems can be split in three steps as shown in Fig. 1: world points are mapped into an oriented projective plane by a conventional $3 \times 4$ projection matrix $\mathbf{P}$; the oriented projective plane is transformed by a non-linear function $\hbar$ (equation 1); the last step is a collineation in the plane $\mathbf{H}_{\mathbf{c}}$ (equation 2). In this case, the function $\ddot{\partial}$ is a linear transformation depending on the camera intrinsics $\mathbf{K}_{\mathbf{c}}$, the relative rotation between the camera and the mirror $\mathbf{R}_{\mathbf{c}}$, and the shape of the reflective surface. Parameters $\xi$ and $\psi$ only depend on the shape of the mirror.

$$
\begin{aligned}
& \mathbf{x}^{\prime}=\hbar(\mathbf{x})=\left(x, y, z+\xi \sqrt{x^{2}+y^{2}+z^{2}}\right)^{t} \\
& \mathbf{x}^{\prime \prime}=\underbrace{\mathbf{K} \mathbf{R}_{\mathbf{c}}\left[\begin{array}{ccc}
\psi-\xi & 0 & 0 \\
0 & \xi-\psi & 0 \\
0 & 0 & 1
\end{array}\right]}_{\mathbf{H}_{\mathbf{c}}} \hbar(\mathbf{x})
\end{aligned}
$$

The non-linear characteristics of the mapping are isolated in $\hbar$ which has a curious geometric interpretation. Since $\mathbf{x}^{\prime}$ is a homogeneous vector representing a point in an oriented projective plane, $\lambda \mathbf{x}^{\prime}$ represents the same point whenever $\lambda>0$ [10]. Assuming $\lambda=1 / \sqrt{x^{2}+y^{2}+z^{2}}$ we obtain from equation 1 that

$$
\left\{\begin{array}{l}
x^{\prime}=\frac{x}{\sqrt{x^{2}+y^{2}+z^{2}}} \\
y^{\prime}=\frac{y}{\sqrt{x^{2}+y^{2}+z^{2}}} \\
z^{\prime}-\xi=\frac{z}{\sqrt{x^{2}+y^{2}+z^{2}}}
\end{array}\right.
$$

Consider $\mathbf{x}$ and $\mathbf{x}^{\prime}$ as projective rays defined in two different coordinates systems with origin in $\mathbf{O}$ (the effective viewpoint) and $\mathbf{O}^{\prime}$. To each projective ray $\mathbf{x}$ going 


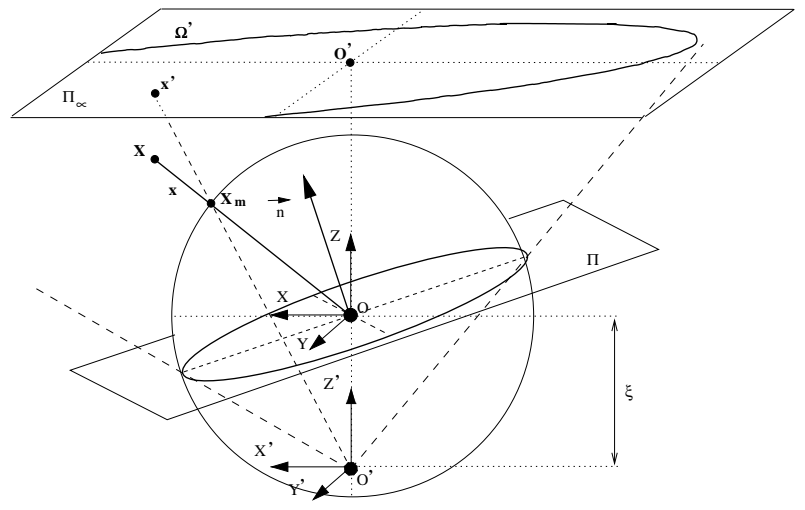

Fig. 2. Sphere model for central catadioptric image formation

through $\mathbf{O}$ corresponds one, and only one, projective ray $\mathbf{x}^{\prime}$ going through $\mathbf{O}^{\prime}$. The correspondence is such that the pencil of projective rays $\mathbf{x}$ intersects the pencil of rays $\mathbf{x}^{\prime}$ in a unit sphere centered in $\mathbf{O}$. The equation of the sphere in the coordinate system with origin in $\mathbf{O}^{\prime}$ is

$$
x^{2}+y^{\prime 2}+\left(z^{\prime}-\xi\right)^{2}=1
$$

We have just derived the well known sphere model shown in Fig. 2 [6]. The homogeneous vector $\mathbf{x}$ can be interpreted as a projective ray joining a 3D point in the scene with the effective projection center $\mathbf{O}$. The projective ray intersects the unit sphere in a single point $\mathbf{X}_{\mathbf{m}}$. Consider a point $\mathbf{O}^{\prime}$ with coordinates $(X, Y, Z)=(0,0,-\xi)^{t}$ where $\xi \in[0,1]$. To each $\mathbf{x}$ corresponds an oriented projective ray $\mathbf{x}^{\prime}$ joining $\mathbf{O}^{\prime}$ with the intersection point $\mathbf{X}_{\mathbf{m}}$ in the sphere surface. The non-linear mapping $\hbar$ corresponds to projecting the scene in the unit sphere and then re-projecting the points on the sphere into a plane from a novel projection center $\mathbf{O}^{\prime}$. Points in the image plane $\mathbf{x}^{\prime \prime}$ are obtained after a collineation $\mathbf{H}_{\mathbf{c}}$ of the $2 \mathrm{D}$ projective points $\mathbf{x}^{\prime}$ (equation 2).

Consider a line in space lying in a plane $\boldsymbol{\Pi}$, with normal $\mathbf{n}=\left(n_{x}, n_{y}, n_{z}\right)^{t}$, which contains the effective viewpoint $\mathbf{O}$ (Fig. 2). The line in the scene is projected into a great circle on the sphere surface. The great circle is obtained by intersecting plane $\boldsymbol{\Pi}$ with the unit sphere. The projective rays $\mathbf{x}^{\prime}$, joining $\mathbf{O}^{\prime}$ with points in the great circle, form a central cone. The central cone, with vertex in $\mathbf{O}^{\prime}$, projects into the conic $\boldsymbol{\Omega}^{\prime}$ in the canonical image plane. As shown in equation 3 , the conic section $\Omega^{\prime}$ is a function of $\mathbf{n}$ and $\xi$ [6]. The catadioptric image of the line is in the conic section $\Omega^{\prime \prime}$ determined by the projective transformation of $\Omega^{\prime}\left(\Omega^{\prime \prime}=\mathbf{H}_{\mathbf{c}}{ }^{-t} \boldsymbol{\Omega}^{\prime} \mathbf{H}_{\mathbf{c}}{ }^{-1}\right)$ [6, 12].

$$
\boldsymbol{\Omega}^{\prime}=\left[\begin{array}{ccc}
n_{x}^{2}\left(1-\xi^{2}\right)-n_{z}^{2} \xi^{2} & n_{x} n_{y}\left(1-\xi^{2}\right) & n_{x} n_{z} \\
n_{x} n_{y}\left(1-\xi^{2}\right) & n_{y}^{2}\left(1-\xi^{2}\right)-n_{z}^{2} \xi^{2} & n_{y} n_{z} \\
n_{x} n_{z} & n_{y} n_{z} & n_{z}^{2}
\end{array}\right]
$$

The re-projection center $\mathbf{O}^{\prime}$ depends only on mirror shape [2]. For a parabolic mirror $\xi=1$ and $\mathbf{O}^{\prime}$ lies in the sphere surface. The re-projection is a stereographic projection. For a hyperbolic mirror $\xi \in(0,1)$ and point $\mathbf{O}^{\prime}$ is inside the sphere in the negative Zaxis. The conventional perspective camera is a degenerate case of central catadioptric projection where $\xi=0$ and $\mathbf{O}^{\prime}$ is coincident with $\mathbf{O}$. 


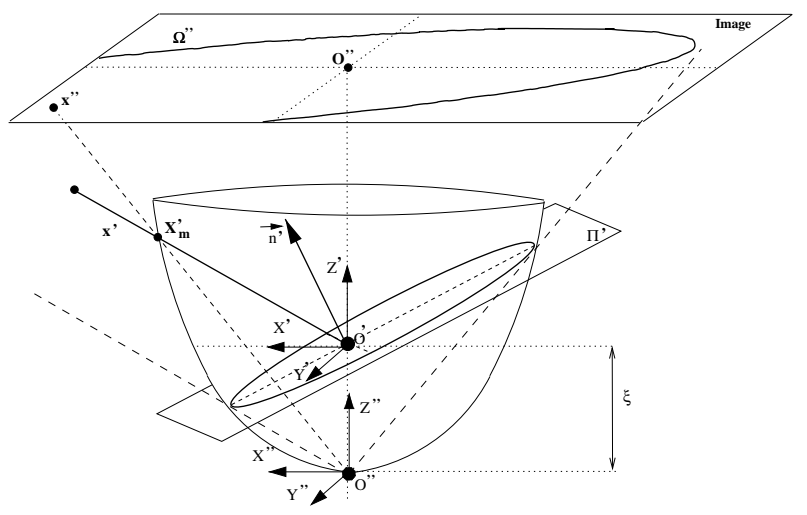

Fig. 3. Paraboloid model for image formation in perspective cameras with lens with radial distortion (division model)

\subsection{Radial Lens Distortion}

In perspective cameras with lens distortion the mapping between points in the scene and points in the world can no longer be described in a linear way. In this paper the radial distortion is modeled using the so called division model [5]. To each point in the scene $\mathbf{X}$ corresponds a projective ray $\mathbf{x}=\mathbf{P X}$ which is transformed into a $2 \mathrm{D}$ projective point $\mathbf{x}^{\prime}=\mathbf{K} \mathbf{x}$ (conventional pin-hole model). Point $\mathbf{x}^{\prime \prime}$, in the final image plane, is related with point $\mathbf{x}^{\prime}$ by a non-linear transformation that models the radial distortion. This transformation, originally introduced in [5], is provided in equation 4, where the radial distortion is parameterized by $\xi$. If $\xi=0$ then points $\mathbf{x}^{\prime}$ and $\mathbf{x}^{\prime \prime}$ are the same, and the camera is modeled by the conventional pin-hole model. Equation 4 corresponds to the inverse of function $\partial$ (see Fig. 1 and Tab. 1), which isolates the nonlinear characteristics of the mapping. Function $\hbar$ is a linear transformation depending on the camera intrinsic parameters $\mathbf{K}$. Notice that the model of equation 4 requires that points $\left[x^{\prime}\right]$ and $\mathbf{x}^{\prime \prime}$ are referenced in a coordinate system with origin in the image distortion center. If the distortion center is not known in advance, we can place it at the image center without significantly affecting the correction [14].

$$
\mathbf{x}^{\prime}=\partial^{-1}\left(\mathbf{x}^{\prime \prime}\right)=\left(x^{\prime \prime} z^{\prime \prime}, y^{\prime \prime} z^{\prime \prime}, z^{\prime \prime 2}+\xi\left(x^{\prime \prime 2}+y^{\prime \prime 2}\right)\right)^{t}
$$

Transformation $\partial$ has a geometric interpretation similar to the sphere model derived for central catadioptric image formation. As stated $\mathbf{x}^{\prime}$ and $\lambda \mathbf{x}^{\prime}$ represent the same point whenever $\lambda$ is a positive scalar. Assuming $\lambda=1 / \sqrt{x^{\prime 2}+y^{\prime 2}}$ in equation 4 yields

$$
\left\{\begin{array}{l}
x^{\prime}=\frac{x^{\prime \prime} z^{\prime \prime}}{x^{\prime \prime 2}+y^{\prime \prime 2}} \\
y^{\prime}=\frac{y^{\prime \prime} z^{\prime \prime}}{x^{\prime \prime 2}+y^{\prime \prime 2}} \\
z^{\prime}-\xi=\frac{z^{\prime \prime 2}}{x^{\prime \prime 2}+y^{\prime \prime 2}} .
\end{array}\right.
$$

Reasoning as in the previous section, $\mathbf{x}^{\prime}$ and $\mathbf{x}^{\prime \prime}$ can be interpreted as projective rays going through two distinct origins $\mathbf{O}^{\prime}$ and $\mathbf{O}^{\prime \prime}$. From equation 5 follows that the two pencils of rays intersect in paraboloid with vertex in $\mathbf{O}^{\prime \prime}$. The equation of this paraboloid in the coordinate system attached to the origin $\mathbf{O}^{\prime}$ is 


$$
x^{2}+y^{\prime 2}-\left(z^{\prime}-\xi\right)=0
$$

An intuitive 'concrete' model for the non-linear transformation $\partial$ (see Tab. 1) is shown in Fig. 3 where the derived parabolic surface is depicted (remind that $\xi \leq 0$ [5]). Consider the projective ray $\mathbf{x}^{\prime}$, going through the camera effective viewpoint $\mathbf{O}^{\prime}$, and intersecting the paraboloid at point $\mathbf{X}_{\mathbf{m}}$. The image point $\mathbf{x}^{\prime \prime}$ is associated with the projective ray joining $\mathbf{X}_{\mathbf{m}}$ with the vertex of the parabola $\mathbf{O}^{\prime \prime}$. The paraboloid of Fig. 3 is a quadric surface in $\wp^{3}$ which is tangent to the plane at infinity on point $\left(X^{\prime}, Y^{\prime}, Z^{\prime}, W^{\prime}\right)^{t}=(0,0,1,0)^{t}$. Thus, if the angle between the projective ray $\mathbf{x}^{\prime}$ and the $Z^{\prime}$ axis is small, the associated point $\mathbf{X}_{\mathbf{m}}$ is close to the plane at infinity and $\mathbf{x}^{\prime}$ and $\mathbf{x}^{\prime \prime}$ are almost coincident. This just shows the well known fact that radial distortion is much more noticeable in the image periphery than in the image center.

Assume a line in the space that, according to the conventional pin-hole model, is projected into a line $\mathbf{n}^{\prime}=\left(n_{x}^{\prime}, n_{y}^{\prime}, n_{z}^{\prime}\right)^{t}$ in the projective plane. Points $\mathbf{x}^{\prime}$, lying on line $\mathbf{n}^{\prime}$, are transformed into image points $\mathbf{x}^{\prime \prime}$ by the non-linear function $\partial$. Since $\mathbf{n}^{\prime t} \mathbf{x}^{\prime}=0$ and $\mathbf{x}^{\prime}=\partial^{-1}\left(\mathbf{x}^{\prime \prime}\right)$, then $\mathbf{n}^{\prime t} \partial^{-1}\left(\mathbf{x}^{\prime \prime}\right)=0$. After some algebraic manipulation the previous equality can be written in the form $\mathrm{x}^{\prime \prime} \Omega^{\prime \prime} \mathrm{x}^{\prime \prime}=0$ with $\Omega^{\prime \prime}$ given by equation 6. The non-linear mapping $\partial$ transforms line $\mathbf{n}^{\prime}$ into a conic section $\Omega^{\prime \prime}$ (see Fig. 3 ).

$$
\Omega^{\prime \prime}=\left[\begin{array}{ccc}
\xi n_{z}^{\prime} & 0 & \frac{n_{x}^{\prime}}{2} \\
0 & \xi n_{z}^{\prime} & \frac{n_{y}^{\prime}}{2} \\
\frac{n_{x}^{\prime}}{2} & \frac{n_{y}^{\prime}}{2} & n_{z}^{\prime}
\end{array}\right]
$$

\section{Embedding $\wp^{2}$ into $\wp^{5}$ Using Veronese Maps}

Perspective projection can be formulated as a transformation of $\Re^{3}$ into $\Re^{2}$. Points $\mathbf{X}=(X, Y, Z)^{t}$ are mapped into points $\mathbf{x}=(x, y)^{t}$ by a non-linear function $f(\mathbf{X})=$ $(X / Z, Y / Z)^{t}$. A standard technique used in algebra to render a nonlinear problem into a linear one is to find an embedding that lifts the problem into a higher dimensional space. For conventional cameras, the additional homogeneous coordinate linearizes the mapping function and simplifies most of the mathematic relations. In the previous section we established a unifying model that includes central catadioptric sensors and lens with radial distortion. Unfortunately the use of an additional homogeneous coordinate does no longer suffice to cope with the non-linearities in the image formation.

In this paper, we propose the embedding of the projective plane into a higher dimensional space in order to study the geometry of general single viewpoint images in a unified framework. This idea has already been explored by other authors to solve other computer vision problems. Higher-dimensional projection matrices are proposed in [15] for the representation of various applications where the world is no longer rigid. In [7], "lifted coordinates" are used to obtain a fundamental matrix between paracatadioptric views. Sturm generalizes this framework and proves the existence of a $3 \times 6$ fundamental matrix between a perspective or affine view, and a catadioptric view [11]. Sturm also shows that, by embedding the projective plane in $\wp^{\mathbf{5}}$, it is possible to obtain a fundamental relation between paracatadioptric views without constraining the camera aspect ratio and skew. 
The present section discusses the embedding of the projective plane $\wp^{2}$ in $\wp^{5}$ using Veronese mapping $[8,9]$. This polynomial embedding preserves homogeneity and is suitable to represent quadratic relations between image points $[4,13]$. Moreover there is a natural duality between lifted points $\widetilde{\mathbf{x}}$ and conics which is advantageous when dealing with catadioptric projection of lines. It is also shown that projective transformations in $\wp^{2}$ can be transposed to $\wp^{5}$ in a straightforward manner.

$$
\mathbf{x} \in \wp^{2} \longrightarrow \widetilde{\mathbf{x}}=\left(x_{0}, x_{1}, x_{2}, x_{3}, x_{4}, x_{5}\right)^{t} \in \wp^{\mathbf{5}}
$$

\subsection{Lifting Point Coordinates}

The following operator $\boldsymbol{\Gamma}$ transforms a pair of $3 \times 1$ vectors $\mathbf{x}, \overline{\mathbf{x}}$ into a $6 \times 1$ vector (equation 7):

$$
\boldsymbol{\Gamma}(\mathbf{x}, \overline{\mathbf{x}})=\left(x \bar{x}, \frac{x \bar{y}+y \bar{x}}{2}, y \bar{y}, \frac{x \bar{x}+z \bar{x}}{2}, \frac{y \bar{z}+z \bar{y}}{2}, z \bar{z}\right)^{t}
$$

Consider that $\mathbf{x}, \overline{\mathbf{x}}$ are the homogeneous coordinates of a pair of points in the projective plane $\wp^{2}$, and that $\widetilde{\mathbf{x}}=\boldsymbol{\Gamma}(\mathbf{x}, \overline{\mathbf{x}})$ is a point in the five dimensional projective space $\wp^{5}$. Function $\Gamma$ maps pairs of points in $\wp^{2}$ into points lying on a primal $\mathbf{S}$ called the cubic symmetroid [8]. $\mathrm{S}$ is a non-linear subset of $\wp^{5}$ defined as follows:

$$
x_{0} x_{2} x_{5}+2 x_{1} x_{3} x_{4}-x_{0} x_{4}^{2}-x_{2} x_{3}^{2}-x_{5} x_{1}^{2}=0, \forall_{\tilde{\mathbf{x}} \in \mathbf{S}}
$$

The second order Veronese mapping used to embed the projective plane into $\wp^{\mathbf{5}}$ is defined as

$$
\mathbf{x} \longrightarrow \widetilde{\mathbf{x}}=\boldsymbol{\Gamma}(\mathbf{x}, \mathbf{x})=\left(x^{2}, x y, y^{2}, x z, y z, z^{2}\right)^{t} .
$$

Notice that the mapping can be directly obtained by assuming $\overline{\mathbf{x}}=\mathbf{x}$ in equation 7 . To each point $\mathbf{x}$, lying in the projective plane, corresponds a point $\widetilde{\mathbf{x}}$ in $\wp^{5}$ such that $\widetilde{\mathbf{x}}=\boldsymbol{\Gamma}(\mathbf{x}, \mathbf{x})$. In this case it can be proved that point $\widetilde{\mathbf{x}}$ verifies the triplet of second order equations provided below.

$$
x_{1}^{2}-x_{0} x_{2}=0 \wedge x_{3}^{2}-x_{0} x_{5}=0 \wedge x_{4}^{2}-x_{2} x_{5}=0, \forall \overline{\mathbf{x}} \in \mathbf{V} .
$$

Equation 10 defines a quadratic surface $\mathbf{V}$ in $\wp^{5}$ known as Veronese surface $[8,9]$.

\subsection{Lifting Lines and Conics}

As discussed in the previous section, a line in the scene is in general projected in a conic when the image is acquired by a central catadioptric system or a camera with radial distortion. A conic in $\wp^{2}$ is represented by a $3 \times 3$ symmetric matrix $\Omega$ or as a $6 \times 1$ homogeneous vector $\widetilde{\omega}$ :

$$
\boldsymbol{\Omega}=\left[\begin{array}{lll}
a & b & d \\
b & c & e \\
d & e & f
\end{array}\right] \longrightarrow \widetilde{\boldsymbol{\omega}}=(a, 2 b, c, 2 d, 2 e, f)^{t}
$$

Accordingly to the unifying theory of section 2, a line in the world might be projected to a degenerate conic like a line whenever the image is acquired by a conventional 
camera $(\xi=0)$ or, whenever the line in the scene is coplanar with the optical axis. Thus, in order to establish a framework to study generalized single viewpoint projection, we must find a suitable way to lift the coordinates of a line $\mathbf{n}$ lying in the projective plane. Since a line is a degenerated conic of rank 1, we can use the mapping of equation 11 with the argument $\Omega=\mathbf{n n}^{t}$. In an equivalent way, but using the operator $\boldsymbol{\Gamma}$, it follows that a line $\mathbf{n}$ in is mapped to $\widetilde{\mathbf{n}}$ such that

$$
\mathbf{n} \rightarrow \widetilde{\mathbf{n}}=\widetilde{\mathbf{D}} \boldsymbol{\Gamma}(\mathbf{n}, \mathbf{n})=\left(n_{x}^{2}, 2 n_{x} n_{y}, n_{y}^{2}, 2 n_{x} n_{z}, 2 n_{y} n_{z}, n_{z}^{2}\right)^{t}
$$

where $\widetilde{\mathbf{D}}$ is a $6 \times 6$ diagonal matrix with diagonal elements $\{1,2,1,2,2,1\}$.

Point $\mathbf{x}$ lies in a conic section $\Omega$ if, and only if, the corresponding lifted point $\widetilde{\mathbf{x}}$ verifies $\widetilde{\boldsymbol{\omega}}^{t} \widetilde{\mathbf{x}}=0$. Moreover, points $\mathbf{x}$ and $\overline{\mathbf{x}}$ are harmonic conjugates with respect to $\boldsymbol{\Omega}\left(\mathbf{x}^{t} \boldsymbol{\Omega} \overline{\mathbf{x}}=0\right)$ if, and only if, $\widetilde{\boldsymbol{\omega}}^{t} \boldsymbol{\Gamma}(\mathbf{x}, \overline{\mathbf{x}})=0$ is verified. In the same way as points and lines are dual entities in $\wp^{2}$, there is a duality between points and conics in $\wp^{5}$. Since the general single viewpoint image of a line is a conic, this duality will prove to be a nice and useful property. As a final remark notice that, while $\wp^{\mathbf{5}}$ is the space of all conics (each point corresponds to a conic), the set of all lines in the plane is mapped into a non-linear subset $\mathbf{V}_{\mathbf{n}}$ that is the projective transformation of the Veronese surface $\mathbf{V}$ by $\widetilde{\mathbf{D}}$ (equation 12 ).

\subsection{Lifting Linear Transformations}

Consider a linear transformation $\mathbf{H}$ in the plane mapping points $\mathbf{x}$ into $\overline{\mathbf{x}}$ such that $\overline{\mathbf{x}}=\mathbf{H} \mathbf{x}$. Points $\mathbf{x}$ and $\overline{\mathbf{x}}$ are lifted into points $\widetilde{\mathbf{x}}$ and $\widetilde{\overline{\mathbf{x}}}$ using the result of equation 9 . It can be proved that, if $\overline{\mathbf{x}}=\mathbf{H x}$ then there is a $6 \times 6$ matrix $\widetilde{\mathbf{H}}$ such that $\widetilde{\overline{\mathbf{x}}}=\widetilde{\mathbf{H}} \widetilde{\mathbf{x}}$. This matrix is provided in equation 13 where $\mathbf{v}_{\mathbf{1}}, \mathbf{v}_{\mathbf{2}}$ and $\mathbf{v}_{\mathbf{3}}$ are the columns of the $3 \times 3$ matrix $\mathbf{H}$.

$$
\mathbf{H}=\left[\begin{array}{c}
\mathbf{v}_{\mathbf{1}}{ }^{t} \\
\mathbf{v}_{\mathbf{2}}{ }^{t} \\
\mathbf{v}_{\mathbf{3}}{ }^{t}
\end{array}\right] \longrightarrow \widetilde{\mathbf{H}}=\left[\begin{array}{l}
\boldsymbol{\Gamma}\left(\mathbf{v}_{\mathbf{1}}, \mathbf{v}_{\mathbf{1}}\right)^{t} \\
\boldsymbol{\Gamma}\left(\mathbf{v}_{\mathbf{1}}, \mathbf{v}_{\mathbf{2}}\right)^{t} \\
\boldsymbol{\Gamma}\left(\mathbf{v}_{\mathbf{2}}, \mathbf{v}_{\mathbf{2}}\right)^{t} \\
\boldsymbol{\Gamma}\left(\mathbf{v}_{\mathbf{1}}, \mathbf{v}_{\mathbf{3}}\right)^{t} \\
\boldsymbol{\Gamma}\left(\mathbf{v}_{\mathbf{2}}, \mathbf{v}_{\mathbf{3}}\right)^{t} \\
\boldsymbol{\Gamma}\left(\mathbf{v}_{\mathbf{3}}, \mathbf{v}_{\mathbf{3}}\right)^{t}
\end{array}\right] \tilde{\mathbf{D}}
$$

The set of linear transformations in $\wp^{2}$ is mapped into a subset of linear transformations in $\wp^{\mathbf{5}}$. Any transformation $\mathbf{H}$, projective or non-projective, has a transformation $\widetilde{\mathbf{H}}$ associated with it. Notice however that there are linear transformations in $\wp^{\mathbf{5}}$ without any correspondence in the projective plane. Equation 14 shows how linear transformations of points and lines/conics in the plane are transposed to the five dimensional space.

$$
\begin{gathered}
\overline{\mathbf{x}}=\mathbf{H x} \quad \longrightarrow \widetilde{\overline{\mathbf{x}}}=\widetilde{\mathbf{H}} \widetilde{\mathbf{x}} \\
\bar{\Omega}=\mathbf{H}^{-t} \boldsymbol{\Omega} \mathbf{H}^{-1} \longrightarrow \widetilde{\bar{\omega}}=\widetilde{\mathbf{H}}^{-t} \widetilde{\boldsymbol{\omega}}
\end{gathered}
$$

Equation 13 defines an operator $\boldsymbol{\Lambda}$ such that $\boldsymbol{\Lambda}(\mathbf{H})=\widetilde{\mathbf{H}}$. It can be proved that

$$
\begin{aligned}
\boldsymbol{\Lambda}\left(\mathbf{H}^{-1}\right) & =\widetilde{\mathbf{H}} \\
\boldsymbol{\Lambda}(\mathbf{H B}) & =\widetilde{\mathbf{H}} \widetilde{\mathbf{B}} \\
\boldsymbol{\Lambda}\left(\mathbf{H}^{t}\right) & =\widetilde{\mathbf{D}} \widetilde{\mathbf{H}}^{t} \widetilde{\mathbf{D}}^{-1} \\
\boldsymbol{\Lambda}\left(\mathbf{I}_{3 \times 3}\right) & =\mathbf{I}_{6 \times 6}
\end{aligned}
$$




\section{The Subset of Line Images}

This section applies the established framework in order to study the properties of line projection in central catadioptric systems and cameras with radial distortion. If it is true that a line is mapped into a conic in the image, it is not true that any conic can be the projection of a line. It is shown that a conic section $\widetilde{\omega}$ is the projection of a line if, and only if, it lies in a certain subset of $\wp^{\mathbf{5}}$ defined by the sensor type and calibration. This subset is a linear subspace for paracatadioptric cameras and cameras with radial distortion, and a quadratic surface for hyperbolic systems.

\subsection{Central Catadioptric Projection of Lines}

Assume that a certain line in the world is projected into a conic section $\Omega^{\prime \prime}$ in the catadioptric image plane. The line lies in plane $\boldsymbol{\Pi}$, depicted in Fig. 2, which contains the projection center $\mathbf{O}$ and is orthogonal to $\mathbf{n}=\left(n_{x}, n_{y}, n_{z}\right)^{t}$. According to section 2, the catadioptric projection of the line is $\Omega^{\prime \prime}=\mathbf{H}_{\mathbf{c}}{ }^{-t} \boldsymbol{\Omega}^{\prime} \mathbf{H}_{\mathbf{c}}{ }^{-1} \cdot \mathbf{H}_{\mathbf{c}}$ is the calibration matrix and $\Omega^{\prime}$ is a conic, provided in equation 3 , which depends on the normal $\mathbf{n}$.

The framework derived in section 3 can be used in order to transpose to $\wp^{\mathbf{5}}$ the model for line projection. Conic $\Omega^{\prime}$ is mapped in $\widetilde{\boldsymbol{\omega}^{\prime}}=\widetilde{\boldsymbol{\Delta}}_{c} \widetilde{\mathbf{n}}$ (equation 16) and the catadioptric image of the line is $\widetilde{\boldsymbol{\omega}^{\prime \prime}}=\widetilde{\mathbf{H}}_{\mathbf{c}} \widetilde{\boldsymbol{\Delta}}_{\mathbf{c}} \widetilde{\mathbf{n}}$. Notice that the linear transformation $\widetilde{\boldsymbol{\Delta}_{\mathbf{c}}}$, derived from equation 3 , does not have an equivalent transformation in the projective plane (equation 13). The catadioptric projection of a line, despite of being non-linear in $\wp^{2}$, is described by a linear relation in $\wp^{5}$. Hence forth, if nothing is said, the collineation $\widetilde{\mathbf{H}_{c}}$ is neglected and it is assumed that $\widetilde{\boldsymbol{\omega}^{\prime}}=\widetilde{\mathbf{H}_{\mathbf{c}}^{-1}} \widetilde{\boldsymbol{\omega}^{\prime \prime}}$ lies in the catadioptric image plane.

$$
\underbrace{\left[\begin{array}{c}
a^{\prime} \\
2 b^{\prime} \\
c^{\prime} \\
2 d^{\prime} \\
2 e^{\prime} \\
f^{\prime}
\end{array}\right]}_{\widetilde{\boldsymbol{\omega}^{\prime}}}=\underbrace{\left[\begin{array}{ccccccc}
1-\xi^{2} & 0 & 0 & 0 & 0 & -\xi^{2} \\
0 & 1-\xi^{2} & 0 & 0 & 0 & 0 \\
0 & 0 & 1-\xi^{2} & 0 & 0 & -\xi^{2} \\
0 & 0 & 0 & 1 & 0 & 0 \\
0 & 0 & 0 & 0 & 1 & 0 \\
0 & 0 & 0 & 0 & 0 & 1
\end{array}\right]}_{\widetilde{\boldsymbol{\Delta}}_{c}} \underbrace{\left[\begin{array}{c}
n_{x}^{2} \\
2 n_{x} n_{y} \\
n_{y}^{2} \\
2 n_{x} n_{z} \\
2 n_{y} n_{z} \\
n_{z}^{2}
\end{array}\right]}_{\widetilde{\boldsymbol{n}}}
$$

As stated in section 3.2 , a line $\mathbf{n}$ in the projective plane is lifted into a point $\widetilde{\mathbf{n}}$ which lies in the quadratic surface $\mathbf{V}_{\mathbf{d}}$. Thus, a generic conic $\widetilde{\boldsymbol{\omega}^{\prime}}$ is the catadioptric projection of a line if, and only if, $\widetilde{\Delta}_{c}^{-1} \cdot \widetilde{\omega^{\prime}} \in \mathbf{V}_{\mathbf{d}} \cdot \mathbf{V}_{\mathbf{d}}$ is the projective transformation of the Veronese surface $\mathbf{V}$ by $\widetilde{\mathbf{D}}$. From equation 10 it follows that, if conic $\widetilde{\boldsymbol{\omega}^{\prime}}=\left(a^{\prime}, 2 b^{\prime}, c^{\prime}, 2 d^{\prime}, 2 e^{\prime}, f^{\prime}\right)^{t}$ is the projection of a line then it must satisfy

$$
\left\{\begin{array}{l}
d^{2}\left(1-\xi^{2}\right)-f^{\prime}\left(a^{\prime}+f^{\prime} \xi^{2}\right)=0 \\
e^{2}\left(1-\xi^{2}\right)-f^{\prime}\left(c^{\prime}+f^{\prime} \xi^{2}\right)=0 \\
b^{\prime 2}-\left(a+f \xi^{2}\right)\left(c^{\prime}+f^{\prime} \xi^{2}\right)=0
\end{array}, \forall \widetilde{\omega^{\prime} \in \zeta}\right.
$$

Equation 17 defines a quadratic surface $\zeta$ in the space of all conics. The constraints of equation 17 have been recently introduced in [16] and used as invariants for calibration purposes. 
The Particular Case of Paracatadioptric Cameras Equation 18 is derived from equation 17 by assuming $\xi=1$. For the particular case of paracatadioptric cameras the subser $\boldsymbol{\zeta}$, defined in $\wp^{5}$, becomes a linear subspace $\varphi$. The conic loci corresponding to paracatadioptric projections of lines lie in a hyperplane $\varphi$ in the space of all conics.

$$
a^{\prime}+f^{\prime}=0 \wedge c^{\prime}+f^{\prime}=0 \wedge b^{2}=0, \forall_{\widetilde{\omega^{\prime} \in \varphi}}
$$

Stating it in a different way, the conic $\widetilde{\omega}$ is a line image if, and only if, it belongs to the null space of matrix $\mathbf{N}_{\mathbf{p}}$

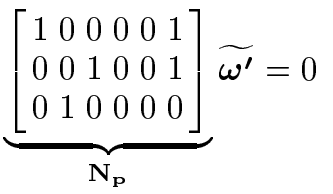

If a point $\mathbf{x}$ belongs to a conic $\Omega$ then $\widetilde{\boldsymbol{\omega}}^{t} \widetilde{\mathbf{x}}=0$ and $\widetilde{\mathbf{x}}$ lies in the prime orthogonal to vector $\widetilde{\boldsymbol{\omega}}[8]$. However, not all points in this prime are lifted coordinates of points in $\wp^{2}$. Points $\mathbf{x}$, lying on $\Omega$, are mapped into a subset of $\wp^{5}$ defined by the intersection of the prime with the Veronese surface $\mathbf{V}$.

Consider the set of all conic sections $\Omega^{\prime}$ where the lines are projected. If there is a point $\mathbf{x}$ common to all elements of the set, then the corresponding point $\widetilde{\mathbf{x}}$ must lie in the intersection of $\mathbf{V}$ with the hyperplane orthogonal to $\varphi$. Points $\widetilde{\mathbf{I}^{\prime}}$ and $\widetilde{\mathbf{J}^{\prime}}$ are computed by intersecting the range of matrix $\mathbf{N}_{\mathbf{p}}{ }^{t}$ with the Veronese surface defined in equation 10. These points are the lifted coordinates of the circular points in the projective plane.

$$
\left\{\begin{array} { l } 
{ \widetilde { \mathbf { I } ^ { \prime } } = ( 1 , i , - 1 , 0 , 0 , 0 ) ^ { t } } \\
{ \widetilde { \mathbf { J } ^ { \prime } } = ( 1 , - i , - 1 , 0 , 0 , 0 ) ^ { t } }
\end{array} \rightarrow \left\{\begin{array}{l}
\mathbf{I}^{\prime}=(1, i, 0)^{t} \\
\mathbf{J}^{\prime}=(1,-i, 0)^{t}
\end{array}\right.\right.
$$

In a similar way, if there is a pair of points $\mathbf{x}, \overline{\mathbf{x}}$ that are harmonic conjugate with respect to all conics $\Omega^{\prime}$ then, the corresponding vector $\Gamma(\mathrm{x}, \overline{\mathrm{x}})$, must be in the intersection of $\mathbf{S}$ with the range of $\mathbf{N}_{\mathbf{p}}{ }^{t}$. The intersection can be determined from equations 8 and 19, defining the cubic symmetroid and matrix $\mathbf{N}_{\mathbf{p}}$. The result is presented in equation 21 where $\lambda$ is a free scalar.

$$
\left\{\begin{array}{l}
\widetilde{\mathbf{P}^{\prime} \mathbf{Q}^{\prime}}=(-\lambda, i, \lambda, 0,0,0)^{t} \rightarrow\left\{\begin{array}{l}
\mathbf{P}^{\prime}=\left(1+\sqrt{1+\lambda^{2}}, \lambda, 0\right)^{t} \\
\mathbf{Q}^{\prime}=\left(1-\sqrt{1+\lambda^{2}}, \lambda, 0\right)^{t}
\end{array}\right. \\
\widetilde{\mathbf{R}^{\prime} \mathbf{T}^{\prime}}=\left(1, \lambda, \lambda^{2}, 0,0,1+\lambda^{2}\right)^{t} \rightarrow\left\{\begin{array}{l}
\mathbf{R}^{\prime}=\left(i, \lambda i, \sqrt{1+\lambda^{2}}\right)^{t} \\
\mathbf{T}^{\prime}=\left(1, \lambda, i \sqrt{1+\lambda^{2}}\right)^{t}
\end{array}\right.
\end{array}\right.
$$

According to equation 20, the paracatadioptric projection of a line must go through the circular points. This is not surprising, since the stereographic projection of a circle is always a circle (see 2). However, not all circles can be the projection of a line. While points $\mathbf{P}^{\prime}, \mathbf{Q}^{\prime}$ are harmonic conjugate with respect to a all circles, the same does not happen with the pair $\mathbf{R}^{\prime}, \mathbf{T}^{\prime}$. Thus, a conic $\Omega^{\prime}$ is the paracatadioptric image of a line if, and only if, it goes through the circular points and satisfies $\mathbf{R}^{\prime} \boldsymbol{\Omega}^{\prime} \mathbf{T}^{\prime}=0$. 


\subsection{Line Projection in Cameras with Radial Distortion}

From section 2.2 we know that a line in the world is mapped into a line $\mathbf{n}^{\prime}$ according to the conventional pin-hole model. The non-linear distortion transforms $\mathbf{n}^{\prime}$ into a conic section $\Omega^{\prime \prime}$ (equation 6). The model for line projection becomes linear when the projective plane is embedded in $\wp^{5}$. Thus, a similar derivation for the catadioptric situation yields

$$
\underbrace{\left[\begin{array}{c}
a^{\prime \prime} \\
2 b^{\prime \prime} \\
c^{\prime \prime} \\
2 d^{\prime \prime} \\
2 e^{\prime \prime} \\
f^{\prime \prime}
\end{array}\right]}_{\widetilde{\omega^{\prime \prime}}}=\underbrace{\left[\begin{array}{cccccc}
0 & 0 & 0 & 0 & 0 & \xi \\
0 & 0 & 0 & 0 & 0 & 0 \\
0 & 0 & 0 & 0 & 0 & \xi \\
0 & 0 & 0 & 0.5 & 0 & 0 \\
0 & 0 & 0 & 0 & 0.5 & 0 \\
0 & 0 & 0 & 0 & 0 & 1
\end{array}\right]}_{\widetilde{\boldsymbol{\Delta}}_{\boldsymbol{d}}} \underbrace{\left[\begin{array}{c}
n_{x}^{\prime 2} \\
2 n_{x}^{\prime} n_{y}^{\prime} \\
n_{y}^{\prime 2} \\
2 n_{x}^{\prime} n_{z}^{\prime} \\
2 n_{y}^{\prime} n_{z}^{\prime} \\
n_{z}^{\prime 2}
\end{array}\right]}_{\widetilde{\boldsymbol{n}^{\prime}}}
$$

Notice that matrix $\widetilde{\boldsymbol{\Delta}}_{\boldsymbol{d}}$ has a structure similar to $\widetilde{\boldsymbol{\Delta}}_{\boldsymbol{c}}$ when the catadioptric system is parabolic ( $\xi=1$ in equation 16$)$. Moreover it can be proved that a conic section $\widetilde{\boldsymbol{\omega}^{\prime \prime}}$ is the distorted projection of a line if, and only if, it lies in a hyperplane $\varsigma$ defined as follows

$$
a^{\prime \prime}-\xi f^{\prime \prime}=0 \wedge c^{\prime \prime}-\xi f^{\prime \prime}=0 \wedge b^{\prime 2}=0, \forall_{\widetilde{\omega^{\prime}} \in \boldsymbol{\varsigma}}
$$

Following a reasoning similar to the previous section, it can be shown that $\Omega^{\prime \prime}$ goes through the circular points of the plane and satisfies $\mathbf{B}^{\prime \prime} \boldsymbol{\Omega}^{\prime \prime} \mathbf{E}^{\prime \prime}=0$ with $\mathbf{B}^{\prime \prime}=$ $\left(i, \lambda i, i \sqrt{\left(1+\lambda^{2}\right) \xi}\right)^{t}$ and $\mathbf{E}^{\prime \prime}=\left(1, \lambda,-\sqrt{\left(1+\lambda^{2}\right) \xi}\right)^{t}$.

\section{Conclusion}

In this paper we studied unifying models for central projection systems and representations of projections of world points and lines. We first proved that the two step projection model through the sphere, equivalent to perspective cameras and all central catadioptric systems, can be extended to cover the division model of radial lens distortion. Having accomodated all central catadioptric as well as radial lens distortion models under one formulation, we established a representation of the resulting image planes in the five-dimensional projective space through the Veronese mapping. In this space, a collineation of the original plane corresponds to a collineation of the lifted space. Projections of lines in the world correspond to points in the lifted space lying in the general case on a quadric surface. However, in the cases of paracatadioptric and radial lens distortions, liftings of the projections of world lines lie on hyperplanes. In ongoing work, we study the epipolar geometry of central camera systems when points are expressed in this lifted space.

\section{Acknowledgments}

The authors are grateful for support through the following grants: NSF-IIS-0083209, NSF-IIS-0121293, NSF-EIA-0324977 and ARO/MURI DAAD19-02-1-0383. 


\section{References}

[1] S. Baker and S. Nayar. A theory of catadioptric image formation. In in Proc. of IEEE International Conference on Computer Vision, Bombay, 1998.

[2] Joao P. Barreto. General Central Projection Systems. Model, Calibration and Visual Servoing. PhD Thesis, University of Coimbra, 2003.

[3] D. C. Brown. Decentering distortion of lens. Photogrametric Engineering, 32(3):444 - 462, 1966.

[4] D. Feldman, T. Padjla, and D. Weinshall. On the epipolar geometry of the crossedslits projection. In Proc. of ICCV'2003 - IEEE Int. Conf. on Computer Vision, Nice, France, October 2003.

[5] A. Fitzgibbon. Simultaneous linear estimation of multipleview geometry and lens distortion. In In Proc. of IEEE Int. Conf. on Computer Vision and Pattern Recognition, Haway, USA, 2001.

[6] C. Geyer and K. Daniilidis. An unifying theory for central panoramic systems and pratical implications. In in Proc. of European Conference on Computer Vision, pages 445 - 461, Dublin, 2000.

[7] C. Geyer and K. Daniilids. Mirrors in motion. epipolar geometry and motion estimation. In Proc. of ICCV'2003 - IEEE Int. Conf. on Computer Vision, Nice, France, October 2003.

[8] J. G. Sample and G. T. Kneebone. Algebraic Projective Geometry. Claredon Press, 1998.

[9] J. G. Sample and L. Roth. Algebraic Geometry. Claredon Press, 1949.

[10] Jorge Stolfi. Oriented Projective Geometry. Academic Press, 1991.

[11] Peter Sturm. Mixing catadioptric and perspective cameras. In in Proc. of IEEE Workshop on Omnidirectional Vision, Copenhagen, Denmark, July 2002.

[12] T. Svoboda and T. Pajdla. Epipolar geometry for central catadioptric cameras. Int. Journal of Computer Vision, 49(1):23-37, August 2002.

[13] R. Vidal, Y. Ma, and S. Sastry. Generalized principal component analysis (gpca). In Proc. of CVPR'2003 - IEEE Int. Conf. on Computer Vision and Pattern Recognition, Madison, Wisconsin, June 2003.

[14] R. Willson and S. Shaffer. What is the center of the image. In In Proc. of IEEE Int. Conf. on Computer Vision and Pattern Recognition, 1993.

[15] L. Wolf and A. Shashua. On projection matrices $\mathbf{P}^{\mathbf{k}} \rightarrow \mathbf{P}^{\mathbf{2}}$, and their applications in computer vision. In Proc. of IEEE Int. Conf. on Computer Vision, 2001.

[16] X. Ying and Z. Hu. Catadioptric camera calibration using geometric invariants. In Proc. of ICCV'2003 - IEEE Int. Conf. on Computer Vision, Nice, France, 2003. 